\title{
Tumor suppressor protein C53 antagonizes checkpoint kinases to promote cyclin-dependent kinase 1 activation
}

\author{
Hai Jiang ${ }^{1,2, *}$, Jianchun $\mathrm{Wu}^{1,{ }^{*}}$, Chen $\mathrm{He}^{1}$, Wending Yang ${ }^{1}$, Honglin $\mathrm{Li}^{1}$ \\ ${ }^{I}$ Children's Memorial Research Center, Feinberg School of Medicine, Northwestern University, Chicago, IL 60614, USA
}

Cyclin-dependent kinase 1 (Cdk1)/cyclin B1 complex is the driving force for mitotic entry, and its activation is tightly regulated by the G2/M checkpoint. We originally reported that a novel protein $\mathbf{C 5 3}$ (also known as Cdk5rap3 and LZAP) potentiates DNA damage-induced cell death by modulating the G2/M checkpoint. More recently, Wang et al. (2007) found that C53/LZAP may function as a tumor suppressor by way of inhibiting NF-kB signaling. We report here the identification of C53 protein as a novel regulator of Cdk1 activation. We found that knockdown of C53 protein causes delayed Cdk1 activation and mitotic entry. During DNA damage response, activation of checkpoint kinase 1 and 2 (Chk1 and Chk2) is partially inhibited by C53 overexpression. Intriguingly, we found that C53 interacts with Chk1 and antagonizes its function. Moreover, a portion of C53 protein is localized at the centrosome, and centrosome-targeting $\mathbf{C 5 3}$ potently promotes local Cdk1 activation. Taken together, our results strongly suggest that C53 is a novel negative regulator of checkpoint response. By counteracting Chk1, C53 promotes Cdk1 activation and mitotic entry in both unperturbed cell-cycle progression and DNA damage response.

Keywords: C53, Cdk1, checkpoint kinases

Cell Research (2009) 19:458-468. doi: 10.1038/cr.2009.14; published online 17 February 2009

\section{Introduction}

Cells utilize checkpoint mechanisms to co-ordinate cell-cycle progression and cellular response to cell cycle irregularities such as replication stress and DNA damage, thereby maintaining accurate transmission of genetic material during cell division. One of the key checkpoints is the $\mathrm{G} 2 / \mathrm{M}$ checkpoint, which regulates activation of Cdk1 (cyclin-dependent kinase 1) and mitotic entry. In response to DNA damage, cells elicit the checkpoint response to either halt cell-cycle progression for DNA repair or induce cell death [1]. Activation of ATM/ATR

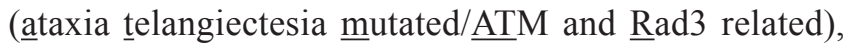
two members of the PI(3)K (phosphatidyl-inositol-3$\mathrm{OH}$ kinase)-like kinases, is the first step for DNA dam-

\footnotetext{
*These two authors contributed equally to this work.

Correspondence: Honglin $\mathrm{Li}$

Tel: +1-773-755-6359; Fax: +1-773-755-6344

E-mail: h-li2@northwestern.edu

${ }^{2}$ Current address: Center for Cancer Research, MIT, 77 Mass. Ave, Cambridge, MA 02139, USA

Received 13 May 2008; revised 29 August 2008; accepted 25 September 2008; published online 17 February 2009
}

age signal transduction $[2,3]$. Locally active ATM/ATR further phosphorylate and activate checkpoint kinases 1 and 2 (Chk1 and Chk2), which rapidly spread the signal to downstream target proteins, such as p53 and Cdc25 phosphatases [4-7]. Phosphorylation and inactivation of Cdc25s by Chk1/Chk2 lead to accumulation of Thr15phosphorylated inactive Cdk1 and the G2/M arrest [4]. In an unperturbed cell cycle, the components of the checkpoints are also essential for surveillance of cellcycle progression. For example, both ATR and Chk1 are essential for animal development and proper cell-cycle progression $[6,8,9]$. Inhibition of Chk1-mediated signaling may lead to increased DNA replication and DNA breakage, aberrant mitotic entry and apoptosis $[6,10]$.

Interestingly, a number of recent studies have demonstrated an important role of the centrosome in regulation of the G2/M transition [11]. The centrosome is the major microtubule-organizing center that contributes to the regulation of cell shape, polarity, adhesion and motility [12]. Recent studies have demonstrated that the centrosome also serves as a solid-phase platform for a multitude of signaling networks, especially cell-cycle control and checkpoint signaling [13]. The Cdk1/cyclin B1 complex accumulates at the centrosome during the interphase, and 
its initial activation occurs at the centrosome in the late prophase [14]. Both positive and negative pathways that regulate Cdk1 activation integrate at the centrosome [11]. Mitotic kinases such as polo-like kinase 1 and aurora-A kinase are localized at the centrosome to promote Cdk1 activation at the onset of mitosis [14-16]. Negative regulators of mitotic entry, such as Chk1 and Chk2, are also reported to localize at the centrosomes $[17,18]$. In the interphase, centrosome-associated Chk1 shields centrosomal Cdk1 from unscheduled activation by Cdc25B, thereby preventing premature mitotic entry [17].

C53 protein (also known as Cdk5rap3 and LZAP) was originally isolated as a binding protein of Cdk5 activator $\mathrm{p} 35$, but its function remains largely elusive $[19,20]$. It is highly conserved during evolution, and its orthologues are found in vertebrate, invertebrate and plants but not in yeast and bacteria. Human C53 consists of 506 amino-acid residues without well-defined domains except for a small region of leucine zipper. Our previ- ous work demonstrated that C53 acts as an important regulator of genotoxin-induced apoptosis [21]. We found that C53 overexpression overrides the G2/M DNA damage checkpoint to promote Cdk1 activation, thereby sensitizing cancer cells to various DNA damage agents [21]. In this study, we further characterized its important role in normal cell-cycle progression and DNA damage response. Intriguingly, we found that C53 interacts and antagonizes checkpoint kinases in both unperturbed cellcycle progression and DNA damage response. Our finding provides a molecular mechanism for C53's chemosensitizing activity, and sheds light on a novel regulatory mechanism for checkpoint kinases and cell-cycle control.

\section{Results}

\section{C53 knockdown delays mitotic entry and Cdk1 activation}

Our previous study suggests that C53 plays an important role in regulation of the G2/M checkpoint in DNA
A

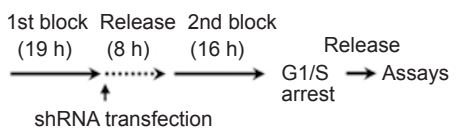

B

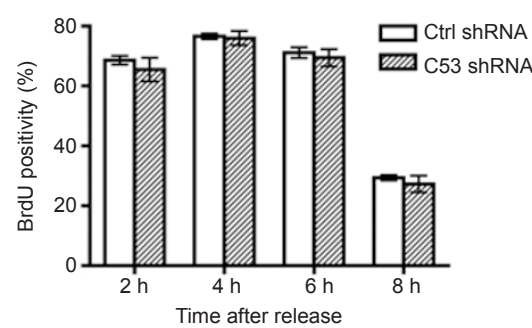

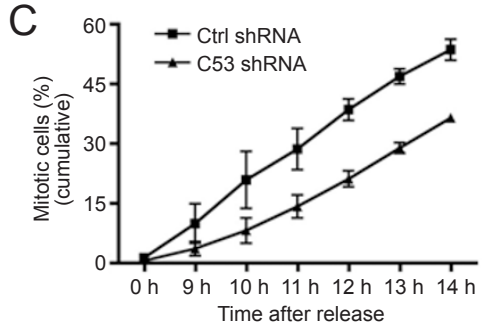

D

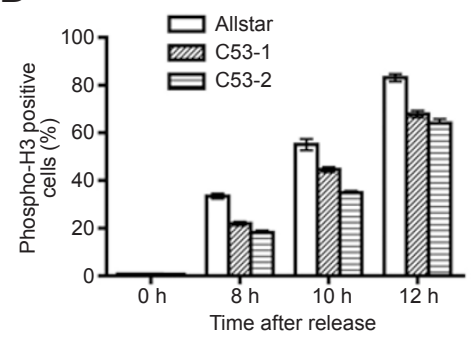

E

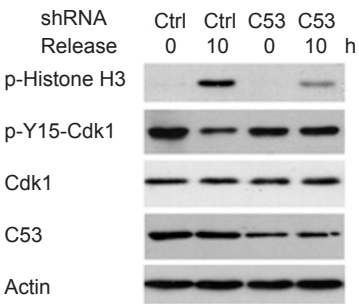

$\mathrm{F}$

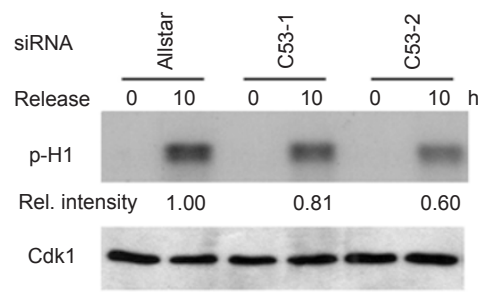

Figure 1 C53 knockdown delays mitotic entry of HeLa cells. (A) Experimental procedure for double-thymidine arrest and C53 knockdown. (B) Knockdown of endogenous C53 did not affect DNA synthesis. HeLa cells were transfected with shRNAs and synchronized at the G1/S transition as described in the methods. Cells were pulse labeled with BrdU (50 $\mu \mathrm{M})$ for 30 min at the indicated time points after release from the second thymidine block. BrdU-positive cells were detected by immunostaining and scored manually. More than 500 cells were counted in each of three independent experiments. (C) shRNA-mediated knockdown of C53 protein delayed mitotic entry. Cell-cycle progression of more than 1000 cells was recorded by time-lapse videomicroscopy. The number of mitotic cells was scored by examination of individual cells. (D) siRNA-mediated C53 depletion led to delayed mitotic entry. Two C53 siRNAs (10 nM) were transfected into HeLa cells by HiPerfect transfection agent. The negative control was Allstar siRNA from Qiagen. Nocodazole $(100 \mathrm{ng} / \mathrm{ml})$ was added to the medium after the second release. Cells were harvested at the indicated time points and subjected to phospho-H3 staining and flow cytometry analysis. (E) C53 knockdown causes accumulation of inactive Cdk1. Cell lysates were collected at the indicated time points after release from the second block, and subjected to immunoblotting using the indicated antibodies. (F) C53 knockdown delayed Cdk1 activation. Cdk1 was precipitated from cell lysates at the indicated time points, and subjected to in vitro kinase assay with histone $\mathrm{H} 1$ as a substrate. The relative intensity was measured with Openlab software and marked at the bottom of the gel. All data are represented as mean \pm SEM. 
damage response. In this study, we attempted to investigate its role in the $\mathrm{G} 2 / \mathrm{M}$ transition during unperturbed cell-cycle progression. Control and C53-knockdown HeLa cells were synchronized at the G1/S boundary by a double-thymidine block, and then released into mitosis. To avoid potential carry-over effects of C53 depletion-induced cell cycle defects in the previous cycle on the mitotic entry during the next cycle, we transfected shRNAs into HeLa cells during the interval between two thymidine blocks, so that we could evaluate the direct impact of C53 knockdown on mitotic entry (Figure 1A) [15]. Expression of endogenous C53 was usually knocked down to more than $50 \%$ after $24 \mathrm{~h}$ by both C53 shRNA and siRNAs (Figure 1E and data not shown). BrdU was added into the medium at the indicated time points to evaluate DNA synthesis. As shown in Figure 1B, incorporation of BrdU into the control and C53-knockdown cells did not differ significantly during cell-cycle progression (Figure 1B), indicating that C53 knockdown may not affect the G1/S transition and DNA synthesis. In contrast, accumulation of mitotic cells was significantly delayed in C53-knockdown cells (Figure 1C). To further examine the specific effect of C53 knockdown on mitotic entry, we repeated this experiment using two C53 siRNAs, and evaluated the mitotic entry using phospho-H3 staining and flow cytometry. As shown in Figure 1D, C53 knockdown by two C53 siRNAs significantly delayed mitotic entry. The variation in mitotic percentage between Figure 1C and 1D may be due to the different toxicities of the transfection methods. Mitotic delay was further confirmed by our observation that there was less phosphorylation of histone $\mathrm{H} 3$ in C53-knockdown cells than in the control cells (Figure 1E, panel of p-histone H3). We also examined whether C53 knockdown delays Cdk1 activation. Consistently, C53-knockdown cells contained more inactive Cdk1 than the control cells did (Figure 1E, panels of Cdk1 and p-Y15-Cdk1). Moreover, in vitro kinase assay showed that Cdk1 in C53-knockdown cells was less active (Figure 1F). Together, our data strongly indicate that C53 may serve as a positive regulator for $\mathrm{Cdk} 1$ activation at the onset of mitosis.

\section{C53 modulates checkpoint kinase-mediated DNA dam- age response}

To further understand how $\mathrm{C} 53$ regulates Cdk1 activation, we took advantage of the experimental system that was used to evaluate the effect of C53 on DNA damage response [21]. We previously demonstrated that C53 overexpression in HeLa cells abolished the G2/M checkpoint-mediated Cdk1 inactivation induced by DNA damage agent etoposide (Etop) [21]. Therefore, we speculated that by dissecting the effect of $\mathrm{C} 53$ on activation of individual components of the DNA damage response pathway, we would identify potential C53 targets. Etop treatment induced the control cells to undergo a prominent G2 arrest, as indicated by the increased number of G2/M cells and enlarged cell body and nuclei (Supplementary information, Figure S1, and data not shown). In agreement with our previous work, C53 overexpression abolished accumulation of G2/M cells induced by Etop, suggesting that $\mathrm{C} 53$ expression abolishes the G2/M arrest induced by Etop treatment (Supplementary information, Figure S1).

We next examined how C53 overexpression influenced checkpoint activation at various molecular levels. As shown in Figure 2, treatment of HeLa cells with DNA damage agents such as Etop and doxorubicin (Dox) and DNA synthesis inhibitor hydroxyurea (HU) caused Cdk1 inactivation, as indicated by the increase in inhibitory phosphorylation of Cdk1 (p-Y15-Cdk1) (Figure 2, panels of p-Y15-Cdk1 and Cdk1, lanes 1, 3, 5 and 7). In agreement with our previous report, C53 overexpression attenuated inhibitory phosphorylation of Cdk1 induced by genotoxic stresses (Figure 2, panels of p-Y15-Cdk1 and

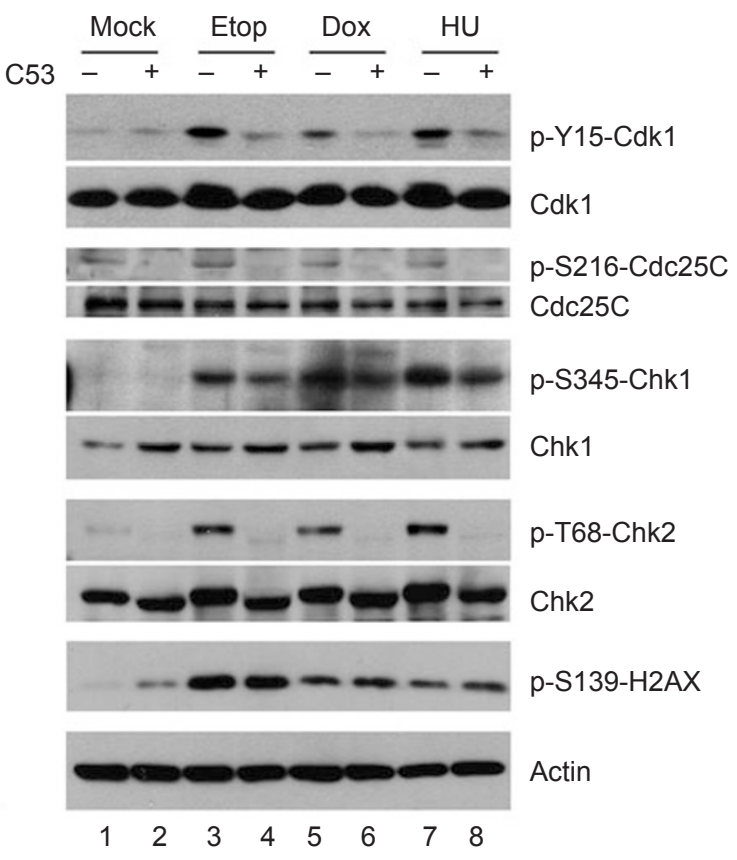

Figure 2 C53 modulates checkpoint kinase-mediated DNA damage response. Ectopic expression of C53 suppressed the DNA damage checkpoint response. HeLa cells were transfected with the C53 or control vectors. At $24 \mathrm{~h}$ after transfection, the cells were treated with etoposide (Etop, $20 \mu \mathrm{M}, 18 \mathrm{~h}$ ), doxorubicin (Dox, $1 \mu \mathrm{M}, 5 \mathrm{~h}$ ) and hydroxyurea (HU, $4 \mathrm{mM}, 18 \mathrm{~h}$ ). Cell lysates were subjected to SDS-PAGE and immunoblotting of indicated antibodies. 
Cdk1, lanes 3-8). Moreover, C53 overexpression blocked inhibitory Ser216 phosphorylation of Cdc25C, the activator of Cdk1 (Figure 2, panels of p-S216-Cdc25C and Cdc25C). As Cdc25C (Ser216) is a bonafide substrate of Chk1 and Chk2 in DNA damage response [4], we further examined the effect of $\mathrm{C} 53$ on activation of Chk1 and Chk2. Intriguingly, C53 overexpression reduced phosphorylation of Chk1 at Ser345 (Figure 2, panels of p-S345-Chk1 and Chk1) as well as Chk2 at Thr68 (Figure 2, panels of p-T68-Chk2 and Chk2), indicating that C53 overexpression may attenuate activation of checkpoint kinases in DNA damage response. We also consistently observed that C53 overexpression slightly increased the protein level of total Chk1 in the presence or absence of DNA damage (Figure 2, panel of Chk1). Active ATM/ ATR are normally responsible for phosphorylation and activation of Chk1 and Chk2. To examine whether C53 overexpression interferes with ATM/ATR activation and activity, we used phosphorylation of histone H2AX (Ser139) as the indicator for ATM/ATR activity [22]. Etop treatment induced a remarkable increase in phosphorylation of $\mathrm{H} 2 \mathrm{AX}$, whereas the effect of doxorubicin and hydroxyurea was moderate (Figure 2, panel of pSer139-H2AX, lanes 1, 3, 5 and 7). C53 overexpression slightly increased $\mathrm{H} 2 \mathrm{AX}$ phosphorylation in the absence of genotoxic stress (Figure 2, panel of p-S139-H2AX, lanes 1 and 2), probably due to a small percentage of cell death induced by C53 overexpression. Importantly, C53 overexpression did not significantly affect elevated $\mathrm{H} 2 \mathrm{AX}$ phosphorylation induced by the genotoxic stresses (Figure 2, compare lanes 4, 6 and 8 with lanes 3, 5 and 7). These results suggest that C53 may act at the level of Chk1/Chk2 in the hierarchy of DNA damage response events.

\section{C53 interacts with Chk1 and antagonizes its activity}

Our finding that C53 negatively influences checkpoint kinase activation may reflect the possible functional interaction between C53 and checkpoint kinases. We first examined whether $\mathrm{C} 53$ interacted directly with Chk1 and Chk2. As shown in Figure 3A, overexpressed Myc-Chk1 and Myc-Chk2 were present in the immunoprecipitate of C53-Flag fusion protein. Additionally, endogenous Chk1 was co-immunoprecipitated with endogenous $\mathrm{C} 53$, but not with control rat IgG (Figure 3B). A doublet of Chk1 may represent different forms of phosphorylated Chk1, as reported previously $[23,24]$. We were unable to detect Chk2 in the same C53 immunoprecipitate (data not shown), probably due to the weak interaction between endogenous C53 and Chk2.

Given the fact that Chk1 is essential for unperturbed cell-cycle progression $[6,8]$, it is likely that C53 exerts its function through modulation of Chk1 activity in an unperturbed cell cycle. To test this hypothesis, we first determined the domain(s) of C53 responsible for C53Chk1 interaction. C53 is cleaved by caspase- 3 at three sites (D268, D282 and D311, our unpublished results and Figure 3F). As shown in Figure 3C, both full-length and the $\mathrm{C}$-terminal fragments $(\mathrm{C} 53 \mathrm{C} 1-\mathrm{C} 3)$ of $\mathrm{C} 53$ protein interacted with Myc-Chk1, while the control protein Bcl2 and the N-terminal fragment of C53 (C53N, residues 1-256) did not (Figure 3C). Among C-terminal cleavage fragments, C53-C1 appeared to bear the highest affinity to Myc-Chk1, indicating that the region between residues 268 and 282 may be important for C53-Chk1 interaction. We also tested the Chk1-C53 interaction in vitro using purified C53 proteins. In agreement with our Co-IP results, GST-C53-C1 pulled down most ${ }^{35}$ S-labeled Chk1 (Supplementary information, Figure S2), which indicates its highest affinity to Chk1. We next tested whether C53 can directly inhibit Chk1 kinase activity. Purified GSTC53 or His-C53 protein did not inhibit GST-Chk1 kinase activity in vitro (data not shown). In HeLa cells, ectopic expression of Myc-Chk1 increased inhibitory phosphorylation of Cdk1 (Figure 3D, panels of Cdk1 and p-Y15$\mathrm{Cdk} 1$, lanes 1 and 2), a result that was in agreement with previous studies [17]. Intriguingly, only C53 and C53C1, but not $\mathrm{C} 53 \mathrm{~N}$, attenuated Chk1-induced inactivation of Cdk1 (Figure 3D, panel of p-Y15-Cdk1, lanes 3-5). Of note, there was more total active Chk1 (indicated by Ser345 phosphorylation) in C53- or C53C1-expressing cells than in $\mathrm{C} 53 \mathrm{~N}$ and control cells (data not shown). Moreover, overexpression of C53 or its derivatives alone did not alter phosphorylation of Cdk1 (Supplementary information, Figure S3). This result suggests that C53 may directly inhibit Chk1 kinase activity in vivo through an activity localized at its C-terminal fragment.

If $\mathrm{C} 53$ indeed negatively regulates Chk1 activity, we speculated that Chk1 inhibition would reverse delayed Cdk1 activation induced by C53 knockdown. Chk1 inhibitor UCN-01 (7-hydroxystaurosporine) (300 nM) was added at $5 \mathrm{~h}$ after the cells were released from the second thymidine block. As shown in Figure 3E, the addition of UCN-01 abolished delayed Cdk1 activation caused by C53 knockdown (Figure 3E, compare lanes 4 and 8 in panels of p-Y15-Cdk1 and p-histone H3). This result indicates that Chk1 activity is indeed responsible for delayed Cdk1 activation in C53-depleted cells at the onset of mitosis, further supporting the notion that $\mathrm{C} 53$ is an antagonist of Chk1.

A portion of C53 is localized at the centrosome and regulates local activation of Cdk1 at the centrosome

To further elucidate the mechanism of C53-mediated 


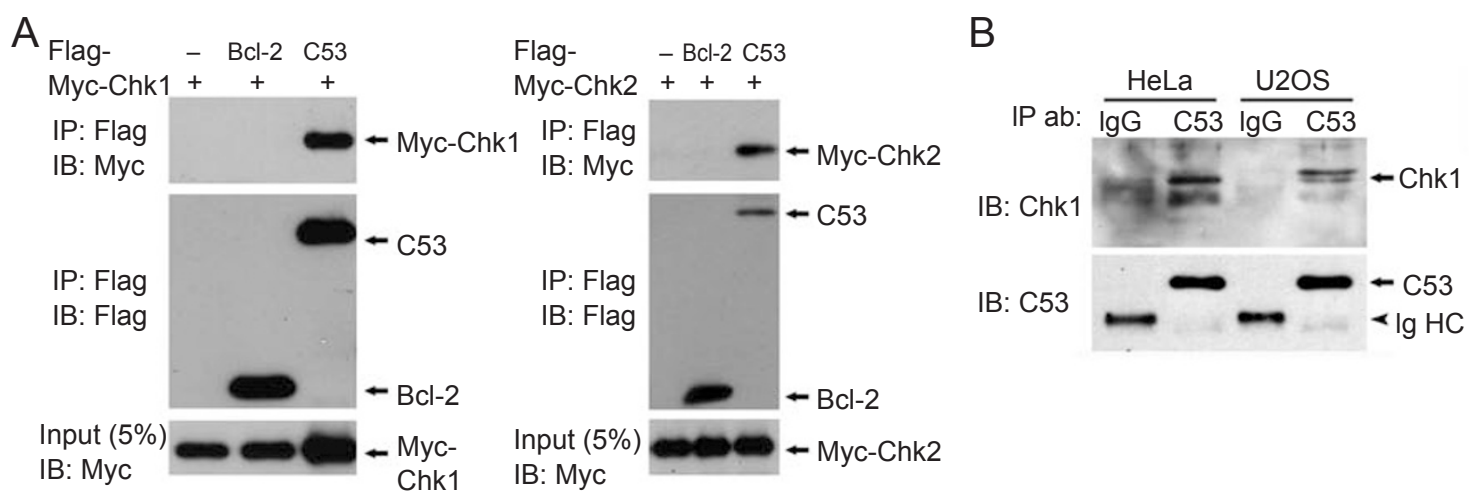

C
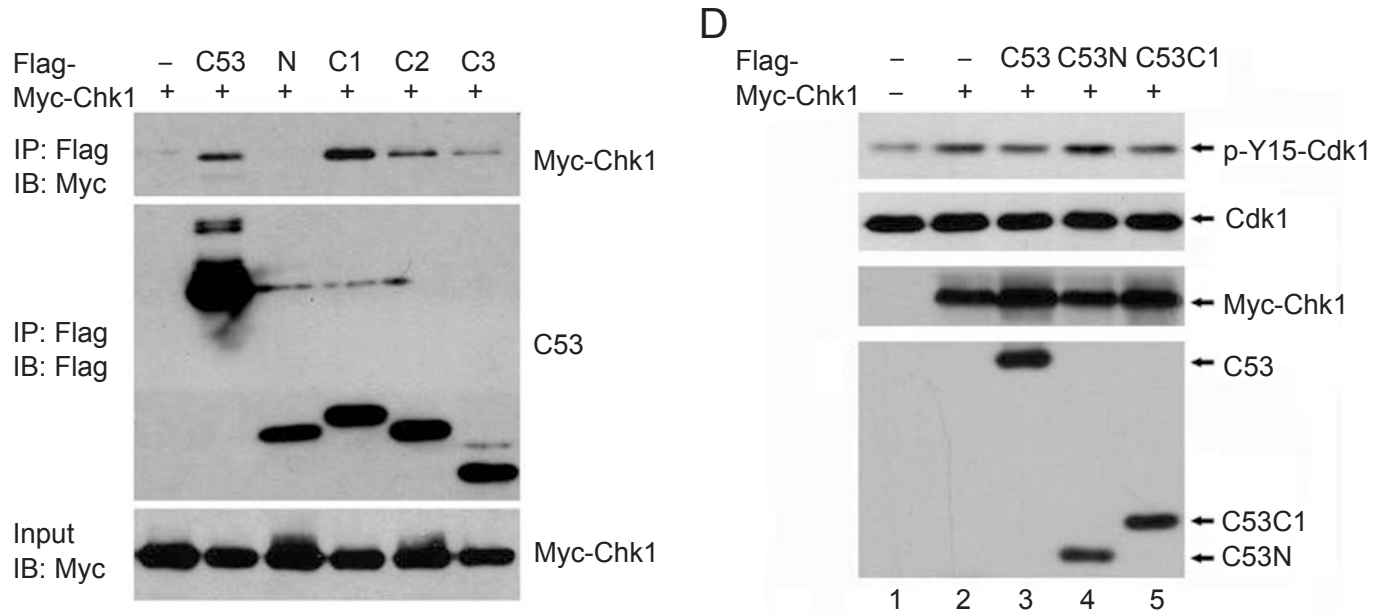

E

UCN-01
shRNA
Release
p-Y15-Cdk1
Cdk1
p-Histone H3

Actin $-\quad-\quad-\quad-\quad-\quad+\quad-\quad+$
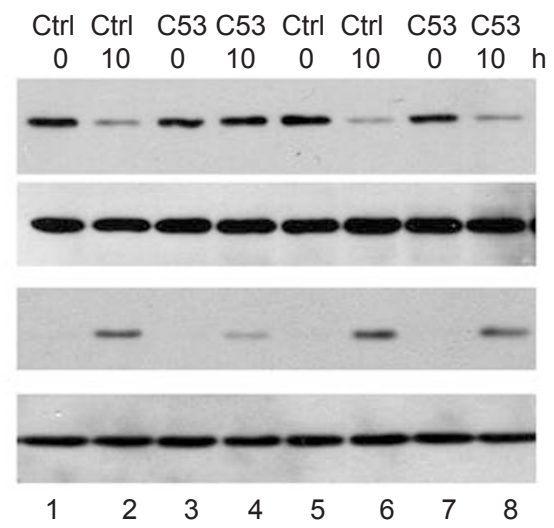

F

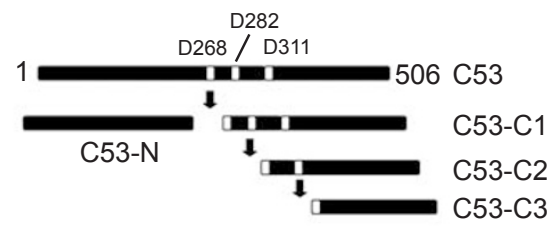

Figure 3 C53 interacts with Chk1 and antagonizes its activity. (A) C53 co-immunoprecipitated with Chk1 and Chk2. Both C53-Flag and Myc-Chk1/Chk2 were overexpressed in HeLa cells. C53-Flag fusion protein was pulled down by Flag (M2) antibody-conjugated agarose beads, and Chk1/Chk2 were detected by immunoblotting using Myc antibody. (B) Co-immunoprecipitation of endogenous C53 and Chk1. Endogenous C53 was pulled down with C53 antibody, and Chk1 was detected by immunoblotting using Chk1 antibody. IgG HC indicated IgG heavy chain. (C) Mapping of C53's Chk1-interacting domains. Myc-Chk1 and C53-Flag and its derivatives were overexpressed in HeLa cells. C53-Flag protein was pulled down by M2conjugated agarose beads. The presence of Chk1 was detected by Myc immunoblotting. (D) Full-length and C-terminal fragment of C53 protein antagonized Chk1-mediated Cdk1 inactivation. HeLa cells were transiently transfected with Myc-Chk1 and C53 constructs. Cells were collected at $24 \mathrm{~h}$ after transfection. Total cell lysates were subjected to immunoblotting using the indicated antibodies. (E) Chk1 inhibitor UCN-01 prevented delayed Cdk1 activation induced by C53 knockdown. HeLa cells were synchronized by a double-thymidine block. UCN-01 (300 nM) was added to the medium at $5 \mathrm{~h}$ after release from the second block. Cells were collected at $10 \mathrm{~h}$ after release, and all the cell lysates were subjected to SDS-PAGE and immunoblotting using the indicated antibodies. (F) C53 constructs used in this study. 


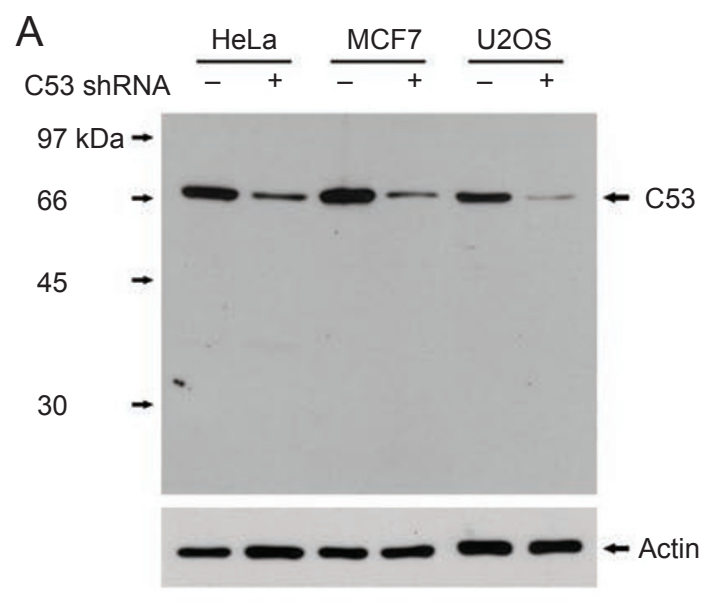

B

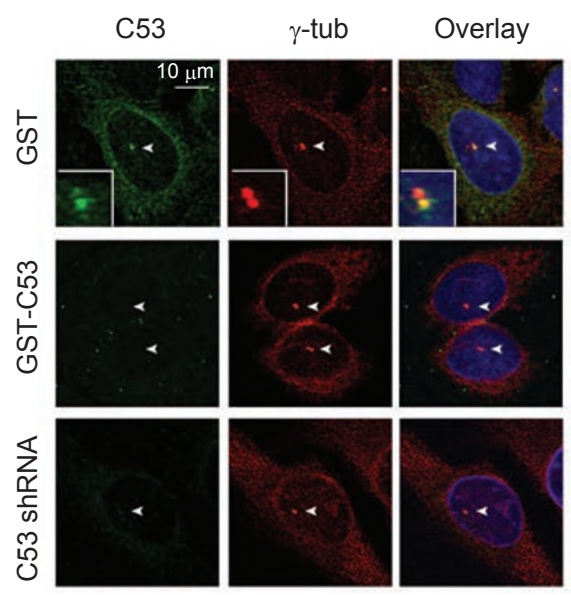

C

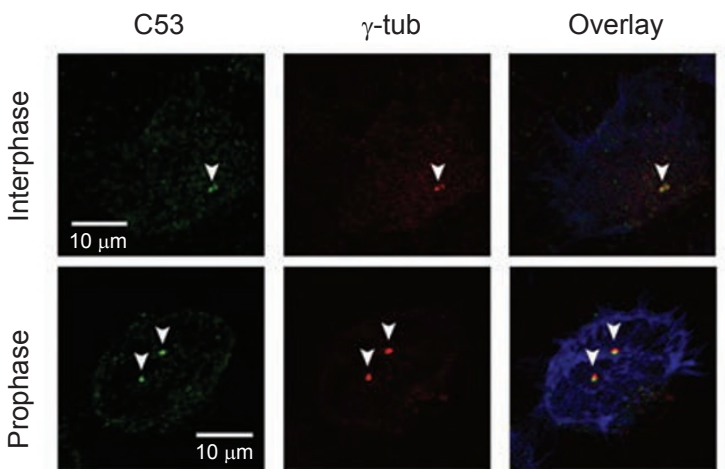

Figure 4 A portion of C53 is localized at the centrosome. (A) Immunoblotting of cell lysates with or without C53 knockdown using purified C53 rat polyclonal antibody. (B) Immunostaining of endogenous C53. U-2OS cells were stained with C53 polyclonal rat antibody and $\gamma$-tubulin monoclonal antibody (GTU-88, Sigma). For depletion, the primary antibodies were incubated with immobilized GST or GST-C53 fusion proteins. For shRNA knockdown, U-2OS cells were transfected with C53 shRNA. After 4-day puromycin selection, the cells were subjected to immunostaining. (C) C53 staining in HeLa cells. To better visualize the centrosomal staining of C53, HeLa cells were pre-extracted with $0.5 \%$ Triton in PBS for 5 min at room temperature. The prophase is indicated by centrosomal separation. All centrosomes are marked by arrowheads. regulation of checkpoint kinases, we raised and affinity purified C53 rat polyclonal antibodies, and examined the subcellular localization of C53. As shown in Figure 4A, our purified polyclonal C53 antibody recognized a single protein with a molecular weight (MW) of 66 $\mathrm{kDa}$, which corresponds to the MW of endogenous C53 protein. C53 is largely localized in the cytoplasm around the nucleus of U-2OS (osteosarcoma) cells (Figure 4B). A portion of C53 is also localized at the centrosomes, as indicated by its co-localization with centrosomal marker $\gamma$-tubulin (Figure 4B). To confirm antibody specificity, we depleted either C53 antibody with immobilized GST-C53 fusion protein, or endogenous C53 with C53 shRNA. Both depletions resulted in negative staining of C53 in the cytoplasm and at the centrosome, suggesting that the antibody staining is C53-specific (Figure 4B). A similar subcellular localization of C53 has also been observed in other cell types, including HeLa cells (Figure 4C), MCF7, DU145 and T47D cells (data not shown). Interestingly, more C53 appeared to be recruited to the centrosome in the prophase (Figure 4C).

\section{C53 regulates local activation of $C d k 1$ at the centrosome}

In an unperturbed cell cycle, the Cdk1/cyclin B1 complex is recruited to the centrosome in the late G2 phase, and initially activated by centrosome-associated Cdc25B at the centrosome in the prophase [14-16]. Centrosomeassociated Chk1 prevents its premature activation in the interphase [17]. Given our findings that a portion of C53 is localized at the centrosomes and that C53 affects the overall activation of Cdk1, we postulated that C53 may also regulate initial activation of $\mathrm{Cdk} 1$ at the centrosome. C53 and Chk1 were co-localized at the centrosome (Supplementary information, Figure S4). We reasoned that if C53 functions as a negative regulator of Chk1, C53 depletion would lead to elevated Chk1 activity, which in turn would cause accumulation of inactive Cdk1 at the centrosome during the G2/M transition. As shown in Supplementary information, Figure S5, a fraction of inactive Cdk1 (p-Y15-Cdk1) was located at the interphase centrosomes of U-2OS cells. In asynchronous U-2OS cells transfected with control shRNA, more than $90 \%$ of the centrosomes displayed C53 staining. In contrast, only $28.9 \%( \pm 5.3 \%)$ of the centrosomes were stained by $\mathrm{C} 53$ antibody in C53 knockdown cells. Interestingly, C53 knockdown led to a higher percentage of cells in which the centrosomes were decorated with inactive $\mathrm{Cdk} 1$ (p-Y15-Cdk1) $(58.5 \% \pm 2.1 \%$ of C53-knockdown cells vs. $27.0 \% \pm 3.0 \%$ of control cells. $P=0.001$ ), indicating that C53 may influence the local activation of Cdk1 (Figure 5A). The level of total inactive Cdk1 was also higher in C53-knockdown cells, as examined by immunoblot- 


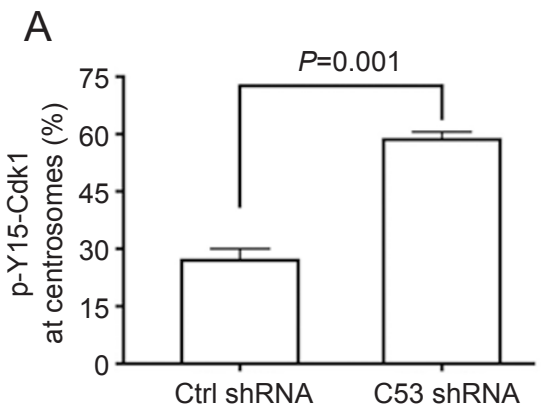

B
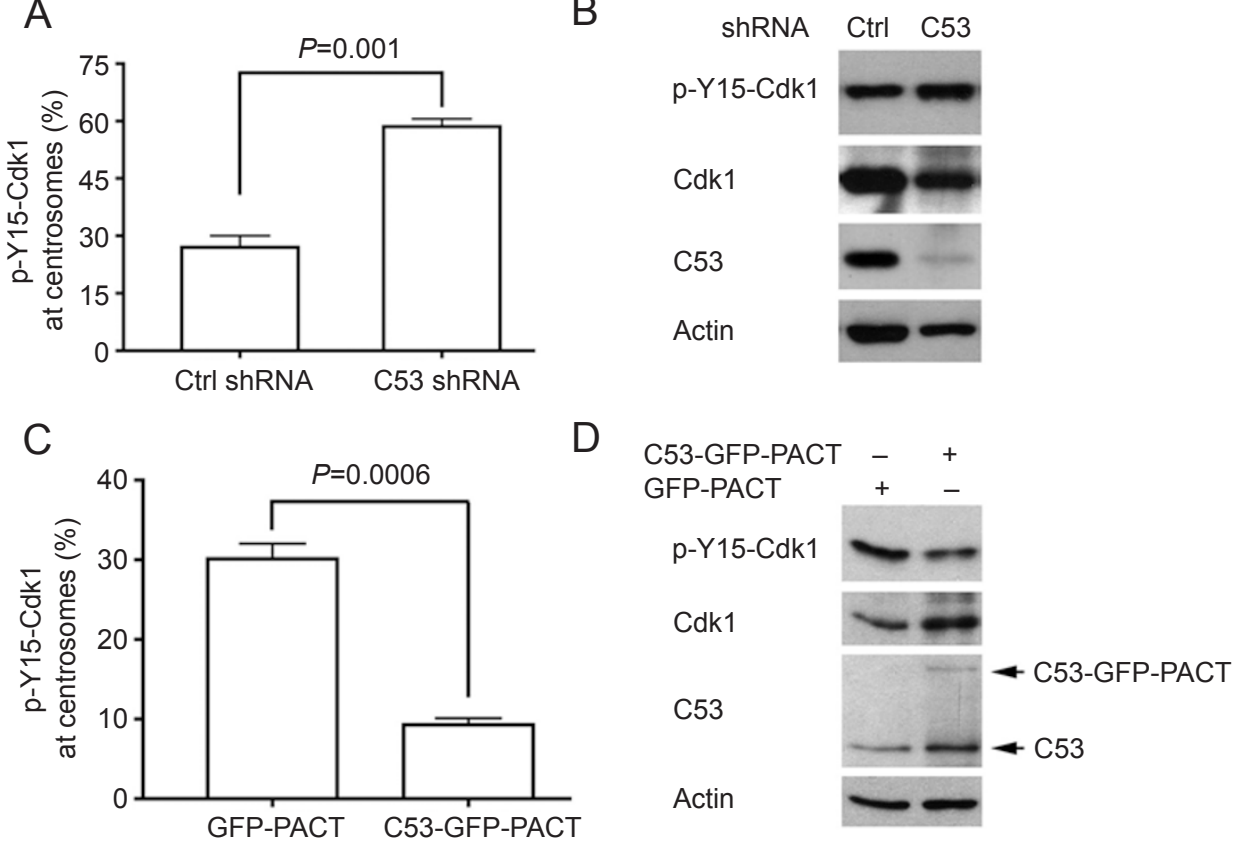

Figure 5 C53 regulates local activation of Cdk1 at the centrosomes. (A) More centrosomes were decorated by inactive Cdk1 in C53-depleted cells. U-2OS cells were infected with retroviruses expressing either control shRNA or C53 shRNA. After a 4-day puromycin selection, U-2OS cells were immunostained with $\mathrm{p}-\mathrm{Y} 15-\mathrm{Cdk} 1$ and $\gamma$-tubulin antibodies. The numbers of cells containing inactive Cdk1-decorated centrosomes were scored manually. More than 200 cells were examined in each of three independent experiments. Data are represented as mean \pm SEM. (B) Total p-Y15-Cdk1 was evaluated by immunoblotting. $\mathrm{U}-2 \mathrm{OS}$ cells were infected with retroviruses expressing control shRNA or C53 shRNA. After a 4-day drug selection (puromycin $2 \mu \mathrm{g} / \mathrm{ml}$ ), cells were collected and subjected to immunoblotting using the indicated antibodies. (C) Centrosome-targeting C53 reduced the number of inactive Cdk1-decorated centrosomes in U-2OS cells. C53-GFP-PACT or GFP-PACT constructs were transfected into U-2OS cells. After $24 \mathrm{~h}$, cells were fixed and immunostained with p-Y15-Cdk1 antibody. p-Y15-Cdk1decorated centrosomes were scored as described above. More than 200 cells were examined in each of three independent experiments. Data are represented as mean \pm SEM. (D) Overexpression of centrosome-targeting C53 promoted overall Cdk1 activation. U-2OS cells (six-well plate) were transfected with C53-GFP-PACT (200 ng) or GFP-PACT construct (200 ng). Cells were collected after $24 \mathrm{~h}$, and subjected to immunoblotting using the indicated antibodies.

ting (Figure 5B).

If $\mathrm{C} 53$ functions as an antagonist of $\mathrm{Chk} 1$, it is conceivable that centrosome-targeting $\mathrm{C} 53$ would counteract centrosomal Chk1 to promote local activation of Cdk1 at the centrosome. We fused the PACT (pericentrinAKAP450 centrosomal targeting) domain of AKAP450 to the C-terminus of C53-GFP protein to generate C53GFP-PACT, a fusion protein that mainly targeted the centrosomes (Supplementary information, Figure S6). As shown in Figure 5C, introduction of C53-GFP-PACT into U-2OS cells remarkably reduced the number of centrosomes decorated by $\mathrm{p}-\mathrm{Y} 15-\mathrm{Cdk} 1(9.3 \% \pm 0.8 \%$ of C53-GFP-PACT-expressing cells vs. $30.1 \% \pm 1.9 \%$ of GFP-PACT-expressing cells, $P=0.0006$ ), suggesting that forced expression of centrosomal C53 is able to antagonize Chk1 and promote Cdk1 activation at the centrosome. Additionally, centrosome-targeting C53-
GFP-PACT was also able to decrease the total pool of p-Y15-Cdk1, as examined by immunoblotting of the total cell lysate (Figure 5D). This result supports the notion that centrosome-associated regulators such as Chk1 are capable of regulating Cdk1 activation both locally and globally [17].

\section{Discussion}

C53 protein (also known as Cdk5rap3 and LZAP) was originally isolated as a Cdk5 activator p35-binding protein in a yeast two-hybrid screening using p35 as the bait protein $[19,20]$, and subsequently identified as a caspase substrate (our unpublished results). More recently, C53 was identified as a binding partner of cAMP response element-binding protein-binding protein [25] and ARF [20]. It is a highly conserved protein, yet its function remains 
largely elusive. We report here that $\mathrm{C} 53$ protein functions as a novel regulator of checkpoint kinases in cell-cycle control. In this study, we demonstrated that C53 acts to antagonize checkpoint kinases to promote $\mathrm{Cdk} 1$ activation. We found that C53 depletion delays Cdk1 activation and mitotic entry in unperturbed cell-cycle progression. Moreover, C53 overexpression partially inhibits checkpoint kinase activation in DNA damage response, thereby overriding the G2/M checkpoint-mediated Cdk1 inactivation. Intriguingly, we found that C53 interacts with Chk1 and counteracts its activity to promote Cdk1 activation both locally and globally. Taken together, our results strongly suggest that C53 acts as a promoter for Cdk1 activation through antagonizing the checkpoint kinases.

Mammalian Chk1 and Chk2 are structurally unrelated, yet functionally overlapping serine/threonine kinases that play critical roles in relaying checkpoint signaling in response to various genotoxic insults [26]. Chk1 is usually activated by ATR-mediated phosphorylation of Ser317 and Ser345 in response to UV and replication stress [6, 27], while Chk2 can be activated mainly by ATM-mediated phosphorylation of its residue Thr68 in response to double-strand DNA breaks [5]. In the absence of DNA damage, Chk2 appears to be largely inactive. In contrast, Chk1 is active even in an unperturbed cell cycle. It is essential for embryonic development and normal cell-cycle progression $[6,8]$. Chk1-deficient embryos display many condensed and fragmented nuclei that apoptose through a p53-independent pathway $[6,8]$. Inhibition of Chk1 activity by its pharmacological inhibitors causes premature Cdk1 activation and mitotic catastrophe [28]. Many proteins have been identified to play important roles in activation of checkpoint kinases, yet inactivation of the checkpoint response remains poorly understood. Several phosphatases have been shown to inactivate checkpoint kinases. Phosphatases PPM1D and PP2 are capable of dephosphorylating Chk1 and Chk2 and inactivating checkpoint response [29-33].

Interestingly, our findings suggest that $\mathrm{C} 53$ may be a novel negative regulator of checkpoint kinases. Our results implicate that Chk1 may be a target of C53 action. First, we found that C53 and Chk1 interact with each other and are co-localized at the centrosomes (Supplementary information, Figure S4). Second, using Cdk1 inactivation as the readout assay for Chk1 intracellular activity, we found that $\mathrm{C} 53$ overexpression antagonizes Myc-Chk1-mediated Cdk1 inactivation (Figure 3D), and that the ability of $\mathrm{C} 53$ fragments to antagonize Chk1 correlated well with their affinity for Chk1 (Figures 3C and 3D). Third, Chk1 inhibition by UCN-01 reversed delayed Cdk1 activation induced by C53 knockdown (Figure 3E).
Finally, forced expression of centrosome-targeting C53 promotes local Cdk1 activation in U-2OS cells (Figure 5), indicating that a centrosomal fraction of $\mathrm{C} 53$ may counteract centrosome-associated Chk1 during mitotic entry.

Nonetheless, the inability of purified C53 to inhibit Chk1 activity in vitro suggests that direct interaction of C53 and Chk1 alone may not be sufficient for C53 antagonizing activity. One possibility is that other protein factors may be needed for C53 function in cells. Another intriguing possibility may come from our observation that a portion of endogenous C53 protein is localized in the centrosome. The centrosome is emerging as an important platform for many signaling pathways [12]. Interestingly, both Chk1 and Chk2 were reportedly found at the centrosomes [17, 18]. Importantly, Kramer et al. demonstrated that centrosome-associated Chk1 shields centrosomal $\mathrm{Cdk} 1$ from unscheduled activation by $\mathrm{Cdc} 25 \mathrm{~B}$, thereby preventing premature mitotic entry [17]. Our result demonstrated that centrosome-targeting $\mathrm{C} 53$ is very potent to promote $\mathrm{Cdk} 1$ activation. Therefore, it is possible that recruitment of $\mathrm{C} 53$ to the centrosome may generate a high concentration of local C53, thereby effectively inhibiting centrosomal Chk1. Consistent with this idea, we observed that more C53 was localized at the prophase centrosomes than in the interphase ones (Figure 4C).

Recently Wang et al. (2007) reported an intriguing finding that LZAP/C53 may function as a tumor suppressor by direct binding and inhibition of RelA, thereby blocking NF- $\kappa \mathrm{B}$ signaling [34]. Our study provides an alternative mechanism of $\mathrm{C} 53$ function, which may also contribute to its tumor suppressor activity. Whether these two mechanisms function in an independent manner or they influence each other remains to be further investigated. Interestingly, we found that C53 localizes at multiple subcellular compartments, including the cytosol, nucleus, centrosome, endoplasmic reticulum and microtubules (our unpublished results), indicating that C53 may be multi-functional.

Recent studies strongly suggest that cell cycle checkpoints are essential for maintenance of replication accuracy and genome stability, and that defects in the checkpoints are largely responsible for tumorigenesis and genome instability of cancer cells [33]. Meanwhile, the DNA damage response network is among the many factors that influence the effectiveness of cancer treatment [35]. Many efforts have been made to identify checkpoint kinase inhibitors as chemo- and radio-sensitizing agents in cancer treatment [36]. Our identification of C53 as a negative regulator of checkpoint kinases sheds light on a novel regulatory mechanism for checkpoint response, and provides a basis for development of novel chemosensitizing agents. 


\section{Materials and Methods}

\section{Tissue culture cells and reagents}

U-2OS cells (from ATCC) were grown in MaCoy's 5A medium supplemented with $10 \%$ fetal bovine serum (FBS), while HeLa, MCF7, T47D and HCT116 and HCT116 (p53-/-) cells were cultured in Dulbecco's modified Eagle's medium (DMEM) supplemented with 10\% FBS and antibiotics. Genotoxic reagents and chemicals were purchased from Sigma.

\section{Construction of C53 expression and shRNA vectors}

Human full-length C53 cDNA and the cDNAs for its truncated mutants (C53N and C53C1-3) were PCR-amplified and subcloned in-frame into pCMV-5a (Sigma), and the resulting constructs were used for expression of C-terminal Flag-tagged C53 in mammalian cells. Primers for full-length C53 were 5'-GCG TCG ACA TGG AGG ACC ATC AGC AC-3' (forward) and 5'-GCG GTA CCC AGA GAG GTT CCC AG-3' (reverse). Primers for C53C1, C2 and $\mathrm{C} 3$ were 5'-GCG TCG ACA TGT GGG GCG GAC TTT GGG GTA G-3' (C1, forward), 5'-GCG TCG ACA TGT CTG GCA TCT CTG CCG AGG-3' (C2, forward) and 5'-GCG TCG ACA TGG ATG CTG TTG CTT TGC AGA T-3' (C3, forward), respectively. The primer for $\mathrm{C} 53 \mathrm{~N}$ was 5 '-CCG GAT CCT TCA ATC GCA TCT TCT GCC AC-3' (reverse). Human cDNAs for Chk1 and Chk2 were amplified from their full-length MGC cDNA clones (ATCC), and subcloned in-frame into pcDNA-Myc (Clontech). Centrosome-targeting C53 (C53-GFP-PACT) was constructed by a two-step PCR. Specifically, C53-GFP cDNA was amplified using specific primers (primer 1: 5'-GCG TCG ACA TGG AGG ACC ATC AGC AC-3' and primer 2: 5'-GGC AAT GAT GGC TTC AAT GTT GGC CTT GTA CAG CTC GTC CAT GCC GAG-3') and pEGFP-N3-C53 as the template, while PACT cDNA was amplified using primers (primer 3: 5'-CTC GGC ATG GAC GAG CTG TAC AAG GCC AAC ATT GAA GCC ATC ATT GCC-3' and primer 4: 5'-GGA TCG ATT TAT GCA CCT TGA TTC AGT CCA AAG CC-3') and GFP-Chk1-PACT (a gift from Dr J Bartek) as the template. Since primers 2 and 3 were complementary, the second PCR was performed using primers 1 and 4 as primers and purified C53GFP and PACT cDNAs as templates. The recombinant C53-GFPPACT cDNA was digested with SalI and ClaI, and subsequently cloned into pLPCX vector (Clontech). All cDNA clones were verified by DNA sequencing.

C53 shRNA vectors were described in Jiang et al. [21]. Retroviruses expressing control shRNA or C53 shRNA were prepared using the 293GP packaging cell line and VSVG envelope protein according to the manufacturer's instructions (BD Clontech).

\section{Cell transfection, cell synchronization and C53 knockdown by ShRNA and SiRNA}

HeLa cells were synchronized by a double-thymidine block. Briefly, HeLa cells were plated at $40 \%$ confluency and arrested with $2 \mu \mathrm{M}$ thymidine. After 19-h incubation, cells were washed four times with fresh medium and transfected with shRNA vectors (C53 and control) using lipofectamine 2000 (Invitrogen). After incubation with DNA-lipid mixture for $3 \mathrm{~h}$, cells were washed twice and incubated in fresh medium for additional $5 \mathrm{~h}$. Subsequently, cells were cultured in medium containing $2 \mu \mathrm{M}$ thymidine and 2 $\mu \mathrm{g} / \mathrm{ml}$ puromycin for the second arrest and drug selection. After a 16-h incubation, cells were released into the cell cycle by incuba- tion in fresh medium. Cells were collected or fixed at the indicated time points and subjected to specific analyses. C53 depletion was also performed using C53 siRNAs that were purchased from Ambion. Negative control siRNA Allstar was obtained from Qiagen. In all, $10 \mathrm{nM}$ of siRNA was used in reverse transfection of $\mathrm{HeLa}$ cells.

For overexpression of C53 and its derivatives, cells were transfected with the expression vectors using either lipofectamine or lipofectamine 2000 (Invitrogen) according to the manufacturer's instructions.

\section{Antibodies, co-immunoprecipitation, immunoblotting and immunofluorescence staining}

Immunoblotting was performed as described [37]. For coimmunoprecipitation assays, cells $\left(4 \times 10^{6}\right)$ were harvested and washed in cold phosphate buffered saline, and then resuspended in $0.5 \mathrm{ml}$ of lysis buffer (20 mM HEPES, pH 7.4, $150 \mathrm{mM} \mathrm{NaCl}$, $5 \mathrm{mM} \mathrm{MgCl}_{2}, 1 \mathrm{mM}$ EDTA, $1 \mathrm{mM}$ DTT, 0.5\% NP-40, $50 \mathrm{mM}$ $\mathrm{NaF}$ and a cocktail of protease inhibitors) for $15 \mathrm{~min}$ on ice. After centrifugation, the supernatant was incubated with the primary antibody for $3 \mathrm{~h}$ at $4{ }^{\circ} \mathrm{C}$, and $10-20 \mu \mathrm{l}$ of ultralink protein $\mathrm{A} / \mathrm{G}$ beads (Pierce) for an additional hour. For immunoprecipitation using anti-Flag M2 antibody, the M2-conjugated agarose beads (Sigma) were used. After three washes in the lysis buffer, the immunoprecipitate was eluted with $0.1 \mathrm{M}$ glycine ( $\mathrm{pH} 2.5$ ), and subsequently subjected to SDS-PAGE and immunoblotting with specific antibodies. For immunofluorescence staining, cells were fixed in $100 \%$ methanol $\left(-20{ }^{\circ} \mathrm{C}\right)$ for $5 \mathrm{~min}$, followed by three washes of PBS. After incubation with blocking buffer (10\% goat serum, $2 \%$ BSA in $1 \times$ PBS) for $1 \mathrm{~h}$ at room temperature, cells were stained with specific primary antibodies overnight at $4{ }^{\circ} \mathrm{C}$. After three washes (5 min each) in PBS, cells were incubated with specific secondary antibodies for $30 \mathrm{~min}$ at room temperature, and subsequently washed in PBS three times ( 5 min each). Cells were mounted in anti-fade reagent Fluosaver (Calbiochem) and analyzed by fluorescence microscopy. Confocal images were acquired using a Zeiss 510 META confocal microscope, while epifluorescence images were obtained using a Leica DMR-HC inverted microscope with Openlab software.

The following antibodies at indicated dilutions were used for immunoblotting: C53 polyclonal rat antibody (affinity purified, 1:1 000 dilution), $\beta$-actin (Sigma, clone AC-15, 1:10 000), Flag (Sigma, M2, 1:1 000), Cdk1 and p-Y15-Cdk1 (Cell Signaling, 1:1 000), Cdc25C (Calbiochem, DCS193, 1:500) and p-S216Cdc25C (Cell Signaling, 1:500), Chk1 (Sigma, DCS-310, 1:3 000) and p-S345-Chk1 (Cell Signaling, 1:1 000), Chk2 (LabVision, DCS-293, 1:1 000) and p-T68-Chk2 (Cell Signaling, 1:1 000), p-S139-H2AX (BioLegend, 1:2 000), p-S10-histone H3 (Upstate, 1:3 000), Myc (Santa Cruz, 9E10, 1:1 000) and $\alpha$-tubulin (Sigma, clone DM 1A, 1:10 000). The following antibodies at the indicated dilutions were used for immunofluorescence staining: C53 (1:200), $\gamma$-tubulin (Sigma, GTU-88, 1:1 000), p-S10-histone H3 (Upstate, 1:500), p-Y15-Cdk1 (Cell Signaling, 1:800) and Chk1 (Sigma, DCS-310, 1:600). All affinity-purified and species-specific fluorophore-conjugated secondary antibodies were obtained from Jackson ImmunoResearch, and used at dilutions between 1:400 and $1: 800$.

BrdU labeling and mitotic index 
BrdU labeling was used to evaluate DNA synthesis. After being released from the second thymidine arrest at the indicated time points, cells grown in 12-well plates were pulse labeled with BrdU $(50 \mu \mathrm{M})$ for $30 \mathrm{~min}$. After three washes of PBS, cells were fixed with $1 \mathrm{ml}$ of Carnoy's fixative (3 parts methanol:1 part glacial acetic acid) at $-20{ }^{\circ} \mathrm{C}$ for $20 \mathrm{~min}$, followed again by three washes of PBS. Subsequently, DNA was denatured by incubation of $2 \mathrm{M}$ $\mathrm{HCl}$ at $37{ }^{\circ} \mathrm{C}$ for $60 \mathrm{~min}$, followed by three washes in borate buffer (0.1 M borate buffer, $\mathrm{pH} 8.5)$. After incubation with the blocking buffer, cells were stained with anti-BrdU antibody (BD Biosciences, 1:100) overnight at $4{ }^{\circ} \mathrm{C}$. After washes in PBS, cells were incubated with Texas Red-conjugated anti-mouse goat IgG for 30 min at room temperature. After the washes, cells were mounted and BrdU-positive cells were manually scored under an immunofluorescence microscope.

Mitotic events were scored by time-lapse videomicroscopy and DNA staining. Cells were synchronized as described above. Realtime images were captured every $10 \mathrm{~min}$ with Openlab software. Mitotic events of control and C53-depleted cells were scored by their morphological changes (from flat to round-up). For each experiment, at least 800 cells of control or C53-depleted cells were videotaped, tracked and analyzed. Alternatively, nocodazole (100 $\mathrm{ng} / \mathrm{ml}$ ) was added into the medium after release, cells were collected, fixed and stained with DNA dye (Hoechst 33258). Mitotic cells were scored by nuclear morphology and DNA condensation. Alternatively, the mitotic index was evaluated by phospho- $\mathrm{H} 3$ staining and flow cytometry.

\section{In vitro kinase assay}

In vitro Cdk1 kinase assay was performed as described in Jiang et al. [21] with minor modification. Cdk1 was immunoprecipitated with Cdk1 antibody (Cell Signaling).

\section{Acknowledgments}

We thank Dr J Bartek (Danish Cancer Society) for Chk1-GFPPACT construct. We are grateful for the insightful advice from Drs Junying Yuan (Harvard Medical School), Qingshen Gao, Jacek Topczewski, Kathy Randell, Bernard Mirkin (Northwestern University) and Marcus Peter (University of Chicago), and the help from Drs Francis Szele and Ed Kang (Children's Memorial Research Center and Northwestern University) on time-lapse videomicroscopy and William Goossens (Children's Memorial Research Center) on confocal microscopy. The project was supported by NIH grant R21 AG027840 and R01 GM081776 (to H Li), Children's Memorial Research Center and the Clarke family.

\section{References}

1 Sancar A, Lindsey-Boltz LA, Unsal-Kacmaz K, Linn S. Molecular mechanisms of mammalian DNA repair and the DNA damage checkpoints. Annu Rev Biochem 2004; 73:39-85.

2 Cimprich KA, Shin TB, Keith CT, Schreiber SL. cDNA cloning and gene mapping of a candidate human cell cycle checkpoint protein. Proc Natl Acad Sci USA 1996; 93:2850-2855.

3 Shiloh Y. Ataxia-telangiectasia and the Nijmegen breakage syndrome: related disorders but genes apart. Annu Rev Genet 1997; 31:635-662.
4 Furnari B, Rhind N, Russell P. Cdc25 mitotic inducer targeted by Chk1 DNA damage checkpoint kinase. Science 1997; 277:1495-1497.

5 Matsuoka S, Huang M, Elledge SJ. Linkage of ATM to cell cycle regulation by the Chk2 protein kinase. Science 1998; 282:1893-1897.

6 Liu Q, Guntuku S, Cui XS, et al. Chk1 is an essential kinase that is regulated by Atr and required for the G(2)/M DNA damage checkpoint. Genes Dev 2000; 14:1448-1459.

7 Shieh SY, Ahn J, Tamai K, Taya Y, Prives C. The human homologs of checkpoint kinases Chk1 and Cds1 (Chk2) phosphorylate p53 at multiple DNA damage-inducible sites. Genes Dev 2000; 14:289-300.

8 Takai H, Tominaga K, Motoyama N, et al.. Aberrant cell cycle checkpoint function and early embryonic death in Chk1(-/-) mice. Genes Dev 2000; 14:1439-1447.

9 Brown EJ, Baltimore D. ATR disruption leads to chromosomal fragmentation and early embryonic lethality. Genes Dev 2000; 14:397-402.

10 Syljuasen RG, Sorensen CS, Hansen LT, et al. Inhibition of human Chk1 causes increased initiation of DNA replication, phosphorylation of ATR targets, and DNA breakage. Mol Cell Biol 2005; 25:3553-3562.

11 Kramer A, Lukas J, Bartek J. Checking out the centrosome. Cell Cycle 2004; 3:1390-1393.

12 Doxsey S, McCollum D, Theurkauf W. Centrosomes in cellular regulation. Annu Rev Cell Dev Biol 2005; 21:411-434.

13 Doxsey S, Zimmerman W, Mikule K. Centrosome control of the cell cycle. Trends Cell Biol 2005; 15:303-311.

14 Jackman M, Lindon C, Nigg EA, Pines J. Active cyclin B1Cdk1 first appears on centrosomes in prophase. Nat Cell Biol 2003; 5:143-148.

15 Hirota T, Kunitoku N, Sasayama T, et al. Aurora-A and an interacting activator, the LIM protein Ajuba, are required for mitotic commitment in human cells. Cell 2003; 114:585-598.

16 Dutertre S, Cazales M, Quaranta M, et al. Phosphorylation of $\mathrm{CDC} 25 \mathrm{~B}$ by aurora-A at the centrosome contributes to the G2-M transition. J Cell Sci 2004; 117:2523-2531.

17 Kramer A, Mailand N, Lukas C, et al. Centrosome-associated Chk1 prevents premature activation of cyclin-B-Cdk1 kinase. Nat Cell Biol 2004; 6:884-891.

18 Tsvetkov L, Xu X, Li J, Stern DF. Polo-like kinase 1 and Chk2 interact and co-localize to centrosomes and the midbody. $J$ Biol Chem 2003; 278:8468-8475.

19 Ching YP, Qi Z, Wang JH. Cloning of three novel neuronal Cdk5 activator binding proteins. Gene 2000; 242:285-294.

20 Wang J, He X, Luo Y, Yarbrough WG. A novel ARF-binding protein (LZAP) alters ARF regulation of HDM2. Biochem $J$ 2006; 393:489-501.

21 Jiang H, Luo S, Li H. Cdk5 activator-binding protein C53 regulates apoptosis induced by genotoxic stress via modulating the G2/M DNA damage checkpoint. J Biol Chem 2005; 280:20651-20659.

22 Ward IM, Chen J. Histone H2AX is phosphorylated in an ATR-dependent manner in response to replicational stress. $J$ Biol Chem 2001; 276:47759-47762.

$23 \mathrm{Ng} \mathrm{CP}$, Lee HC, Ho CW, et al. Differential mode of regulation of the checkpoint kinases CHK1 and CHK2 by their regulatory domains. J Biol Chem 2004; 279:8808-8819. 
24 Shiromizu T, Goto H, Tomono Y, et al. Regulation of mitotic function of Chk1 through phosphorylation at novel sites by cyclin-dependent kinase 1 (Cdk1). Genes Cells 2006; 11:477485.

25 Yin X, Warner DR, Roberts EA, Pisano MM, Greene RM. Novel interaction between nuclear co-activator CBP and the CDK5 activator binding protein - C53. Int J Mol Med 2005; 16:251-256.

26 Bartek J, Lukas J. Chk1 and Chk2 kinases in checkpoint control and cancer. Cancer Cell 2003; 3:421-429.

27 Guo Z, Kumagai A, Wang SX, Dunphy WG. Requirement for Atr in phosphorylation of Chk1 and cell cycle regulation in response to DNA replication blocks and UV-damaged DNA in Xenopus egg extracts. Genes Dev 2000; 14:2745-2756.

28 Niida H, Tsuge S, Katsuno Y, Konishi A, Takeda N, Nakanishi M. Depletion of Chk1 leads to premature activation of Cdc2-cyclin B and mitotic catastrophe. J Biol Chem 2005; 280:39246-39252.

29 Lu X, Nannenga B, Donehower LA. PPM1D dephosphorylates Chk1 and p53 and abrogates cell cycle checkpoints. Genes Dev 2005; 19:1162-1174.

30 Fujimoto H, Onishi N, Kato N, et al. Regulation of the antion- cogenic Chk2 kinase by the oncogenic Wip1 phosphatase. Cell Death Differ 2006; 13:1170-1180.

31 Oliva-Trastoy M, Berthonaud V, Chevalier A, et al. The Wip1 phosphatase (PPM1D) antagonizes activation of the Chk2 tumour suppressor kinase. Oncogene 2007; 26:1449-1458.

32 Dozier C, Bonyadi M, Baricault L, Tonasso L, Darbon JM. Regulation of Chk2 phosphorylation by interaction with protein phosphatase 2A via its B' regulatory subunit. Biol Cell 2004; 96:509-517.

33 Nojima H. G1 and S-phase checkpoints, chromosome instability, and cancer. Methods Mol Biol 2004; 280:3-49.

34 Wang J, An H, Mayo MW, Baldwin AS, Yarbrough WG. LZAP, a putative tumor suppressor, selectively inhibits NFkappaB. Cancer Cell 2007; 12:239-251.

35 Zhou BB, SJ Elledge. The DNA damage response: putting checkpoints in perspective. Nature 2000; 408:433-439.

36 Zhou BB, Bartek J. Targeting the checkpoint kinases: chemosensitization versus chemoprotection. Nat Rev Cancer 2004; 4:216-225.

37 Li H, Bergeron L, Cryns V, et al. Activation of caspase-2 in apoptosis. J Biol Chem 1997; 272:21010-21017.

(Supplementary information is linked to the online version of the paper on the Cell Research website.) 